\title{
Instability of Water Jet: Aerodynamically Induced Acoustic and Capillary Waves ${ }^{1}$
}

\author{
Göran I. Broman ${ }^{a}$ and Oleg V. Rudenko ${ }^{a, b, c, d}$ \\ ${ }^{a}$ School of Engineering, Blekinge Institute of Technology, Karlskrona, SE-37179 Sweden \\ ${ }^{b}$ Department of Physics, M.V. Lomonosov Moscow State University, Moscow, 119991 Russia \\ ${ }^{c}$ Schmidt Earth Physics Institute of Russian Academy of Sciences, Moscow, Russia \\ ${ }^{d}$ Prokhorov General Physics Institute of Russian Academy of Sciencess, Moscow, Russia \\ e-mail: rudenko@acs366.phys.msu.ru;goran.broman@bth.se
}

Received October 20, 2011

\begin{abstract}
High-speed water jet cutting has important industrial applications. To further improve the cutting performance it is critical to understand the theory behind the onset of instability of the jet. In this paper, instability of a water jet flowing out from a nozzle into ambient air is studied. Capillary forces and compressibility of the liquid caused by gas bubbles are taken into account, since these factors have shown to be important in previous experimental studies. A new dispersion equation, generalizing the analogous Rayleigh equation, is derived. It is shown how instability develops because of aerodynamic forces that appear at the streamlining of an initial irregularity of the equilibrium shape of the cross-section of the jet and how instability increases with increased concentration of gas bubbles. It is also shown how resonance phenomena are responsible for strong instability. On the basis of the theoretical explanations given, conditions for stable operation are indicated.
\end{abstract}

Keywords: jet, instability, acoustics, capillary wave, airflow, resonance

DOI: $10.1134 / \mathrm{S} 1063771012050053$

\section{INTRODUCTION}

Material processing based on high-speed liquid jet flow has found widespread applications. The operation principle can be described in the following way. The working fluid is pressed to hundreds of MPa. Thereafter the liquid is channeled through a narrow nozzle and flows out at speed of hundreds of $\mathrm{m} / \mathrm{s}$. The kinetic energy of the jet and the resulting braking pressure near the target are very high. As a result, the jet is capable to cut many types materials. With an abrasive mixed into the jet, it can cut materials of any strength (such as super hard alloys, composites, ceramics and rock). Water jet machining is used also for "milling" and surface cleaning. Advantages include clean cut edges (which minimizes the need for post-operations), high accuracy and flexibility of produced shapes, small material losses, negligible thermal influence on the work piece (which minimizes the risk of oxidation, ignition, distortion and material degradation) and inert process materials (mainly water and sand). All of this contributes to its potential of being an effective technology that can support sustainable development of industry and society.

With these advantages, water jet machining is used also for combustible materials and it has shown to be effective for the decommissioning of nuclear power

\footnotetext{
${ }^{1}$ The article is published in the original.
}

plants. It will likely be used extensively at the Ignalina nuclear power plant in Lithuania. This is the first plant that will be completely dismantled.

Water jet machining is of importance also in medicine, in particular, for cutting of bones and soft tissues. For example, water jet is used for preparation of bone implants and treatment for inter vertebral rupture (socalled hydro discectomy) was developed recently. Replacing the scalpel by the water jet instrument offers the possibility to perform the surgical operation without general anesthesia (http://mst.ru/products/ spine/treatment/hydrocision/).

Studies of the water jet technology were initiated in the late 1950s to the early 1960s in USA and in former USSR. In the 1970s the technology was developed in USA, combining a drill with the water jet. In 1972 Professor Norman Franz of Michigan University worked with McCartney Manufacturing Company to install the first industrial water jet cutter. The equipment was installed in Alton Boxboard. Flow Industries began to market water jet cutting equipment. It was Flow Industries who added sand to a pressurized cleaning system. It was then demonstrated that abrasive water jet could cut through metals and ceramics. From here on the water jet cutting industry took off (http://www. mfg.mtu.edu/cyberman/machining/non_trad/waterjet/ \#History). Today there are many companies manufacturing such equipment, e.g., Flow International Sys- 


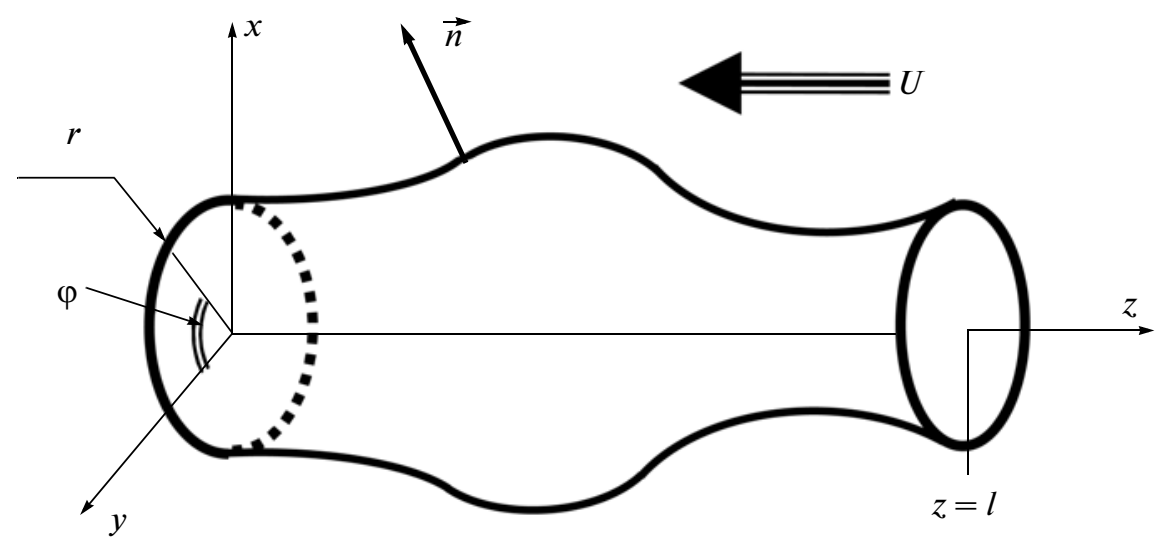

Fig. 1. Cylindrical coordinates attached to the water jet.

tems (USA), OMAX (USA), ANT (Germany) and Water Jet Sweden (Sweden).

To further improve the cutting performance it is critical to understand the theory behind the onset of instability of the jet. The aim of this paper is to study how some important factors influence the stability of a high-speed water jet flowing out from a nozzle into ambient air and to find conditions for the onset of instability.

\section{SOME PHENOMENA RESPONSIBLE FOR INSTABILITY}

As said above, the idea of water jet cutting was suggested long ago (for a review, see [1]). Pressures of 200-500 MPa and exit velocities of 200-1000 m/s are common in modern machines.

Such high-speed jets are sensitive to external disturbances such as fluctuation of pressure or velocity at the input of the nozzle, sound waves excited by vibration of the work piece, capillary waves at the surface of the jet and other irregularities of the surface interacting with the surrounding air. These disturbances gain in strength with increased distance from nozzle along the jet. As a result, the jet widens, brakes and even breaks into droplets. These transformations lead to an almost complete loss of cutting capacity.

The stability of a low-speed liquid jet in air has been the subject of studies for more than 100 years. The pioneering study was performed by Rayleigh [2, 3]. Many papers and books [4-6] are devoted to liquid jets in liquids (submerged jets) and to gaseous jets in gases. High-speed liquid jets in gases are studied much less.

To better understand the onset of instability, the spatial increment of initial irregularities along the jet must be calculated. This increment determines the maximum length at which the jet is laminar and keeps its diameter comparable to the diameter of the output cross-section of the nozzle. Consideration must be given, first of all, to the following physical factors: (i) capillary forces at the free surface and (ii) aerody- namic forces appearing at the streamlining of jet irregularities.

\section{MATHEMATICAL MODEL ALLOWING FOR CAPILLARY FORCES}

In this section a mathematical description of surface tension for a cylindrical jet is given. Note, that the first section of the derivation of basic equations is not original (see. [3, 4]). It is described here for better understanding of subsequent new generalizations.

Cylindrical coordinates $r, z, \varphi$ are used (see Fig. 1). The axial coordinate $z$ coincides with the axis of the jet. The free surface is described by the function $r=$ $r(z, \varphi)$.

The area of this surface is given by the integral $[3,4]$ :

$$
f=\int_{0}^{l} d z \int_{0}^{2 \pi}\left[1+\left(\frac{\partial r}{\partial z}\right)^{2}+\left(\frac{1}{r} \frac{\partial r}{\partial \varphi}\right)^{2}\right]^{1 / 2} r d \varphi .
$$

The equilibrium (undisturbed) shape of the surface is given by $r=R$, where $R$ is the radius of a concentric cylinder. The disturbed surface is described by the function $r=R+\varsigma(z, \varphi)$, where $\varsigma(z, \varphi)$ is the displacement, which is assumed to be small in comparison to $R$.

Series expansion of the sub-integral function in (15) with account for small terms of order of $\varsigma$ and $\varsigma^{2}$ transforms the integral into:

$$
f=\int_{0}^{l} \int_{0}^{2 \pi}\left[(R+\varsigma)+\frac{R}{2}\left(\frac{\partial \zeta}{\partial z}\right)^{2}+\frac{1}{2 R}\left(\frac{\partial \varsigma}{\partial \varphi}\right)^{2}\right] d z d \varphi .
$$

The variation of the surface area caused by a variation of the displacement $\varsigma$ becomes:

$$
\delta f=\iint\left[\delta \varsigma+R \frac{\partial \varsigma}{\partial z} \frac{\partial}{\partial z} \delta \varsigma+\frac{1}{R} \frac{\partial \varsigma}{\partial \varphi} \frac{\partial}{\partial \varphi} \delta \varsigma\right] d z d \varphi .
$$

Assuming zero variation $(\delta \varsigma=0)$ at both ends $(z=0$, and $z=1)$ and a cyclic character of the variation $\delta \zeta$ 
with respect to the polar angle $\varphi$, integration by parts of the second and third terms in (16) give:

$$
\delta f=\int_{0}^{l} \int_{0}^{2 \pi}\left[1-R \frac{\partial^{2} \varsigma}{\partial z^{2}}-\frac{1}{R} \frac{\partial^{2} \varsigma}{\partial \varphi^{2}}\right] \delta \varsigma d z d \varphi .
$$

On the other hand, the variation of the surface can also be expressed through the main radii of curvature $\left(R_{1}\right.$, $R_{2}$ ) of the surface at a given point as:

$$
\delta f=\int_{0}^{l} \int_{0}^{2 \pi}\left[\frac{1}{R_{1}}+\frac{1}{R_{2}}\right] \delta \varsigma r d z d \phi .
$$

Combining (17) and (18), also considering that $r \approx$ $R+\varsigma$, gives:

$$
\left(\frac{1}{R_{1}}+\frac{1}{R_{2}}\right)=\frac{1}{R}-\frac{\varsigma}{R^{2}}-\frac{\partial^{2} \varsigma}{\partial z^{2}}-\frac{1}{R^{2}} \frac{\partial^{2} \varsigma}{\partial \varphi^{2}} .
$$

At equilibrium, when the jet surface has the form of a concentric cylinder $\left(\varsigma=0, R_{2}=\infty\right)$, the evident result $R_{1} \equiv R$ follows from (19).

Now it is possible to calculate the pressure inside the jet caused by the surface tension. Using Laplace's formula and equation (19) gives:

$$
\begin{gathered}
p^{\prime}=p_{0}+\sigma\left(\frac{1}{R_{1}}+\frac{1}{R_{2}}\right) \\
=p_{0}+\sigma\left(\frac{1}{R}-\frac{\varsigma}{R^{2}}-\frac{\partial^{2} \varsigma}{\partial z^{2}}-\frac{1}{R^{2}} \frac{\partial^{2} \varsigma}{\partial \varphi^{2}}\right) .
\end{gathered}
$$

This formula connects the difference in pressure when crossing the surface and the main radii of the surface curvature. Here $\sigma$ is the coefficient of surface tension.

It is convenient now to calculate the time derivative of (20):

$$
\frac{\partial p^{\prime}}{\partial t}+\sigma\left(\frac{1}{R^{2}} u+\frac{\partial^{2} u}{\partial z^{2}}+\frac{1}{R^{2}} \frac{\partial^{2} u}{\partial \varphi^{2}}\right)=0, \quad u=\frac{\partial \varsigma}{\partial t},
$$

and shift variables using the velocity potential $\Phi$ :

$$
u=\frac{\partial \Phi}{\partial r}, \quad p^{\prime}=-\rho_{L} \frac{\partial \Phi}{\partial t} .
$$

Here, $\rho_{L}$ is the density of the liquid. From this, the following important equation is derived:

$$
\rho_{L} \frac{\partial^{2} \Phi}{\partial t^{2}}-\frac{\sigma}{R^{2}} \frac{\partial}{\partial r}\left(\Phi+\frac{\partial^{2} \Phi}{\partial \varphi^{2}}+R^{2} \frac{\partial^{2} \Phi}{\partial z^{2}}\right)=0 .
$$

Equation (22) is valid at the surface of the cylinder $(r=R)$. Therefore, (22) can be named a "dynamic boundary condition".

In the volume of water, the Laplace equation for the velocity potential must be fulfilled:

$$
\Delta \Phi=\frac{\partial^{2} \Phi}{\partial z^{2}}+\frac{1}{r^{2}} \frac{\partial^{2} \Phi}{\partial \varphi^{2}}+\frac{1}{r} \frac{\partial}{\partial r}\left(r \frac{\partial \Phi}{\partial r}\right)=0 .
$$

The use of (23), valid for an incompressible liquid, is possible when the disturbances propagate along the jet with velocities much smaller than the speed of sound in water.

Sometimes, small air bubbles can appear in the liquid, e.g., because of their adhesion to abrasive parti- cles. The resulting compressibility of the fluid can considerably influence the behaviour of the jet and must therefore also be considered. It is known, that the compressibility of such a gas-liquid mixture is much higher than the compressibility of pure water. The velocity of sound is given by the Mallock [7] formula:

$$
c_{e f f}=c_{A} \sqrt{\frac{\rho_{A}}{\rho_{L} v}}
$$

Here, $v$ is the volume concentration of bubbles (which is supposed to be small) and $\rho_{A}$ and $\rho_{L}$ are the densities of air and water, respectively. At $v=10^{-3}$ the effective velocity of sound in an air-water mixture is about the same as the velocity of sound in pure air. If $v=0.5, c_{\text {eff }}$ falls down to its minimum value of $23.8 \mathrm{~m} / \mathrm{s}$. For such strongly air-saturated water, the Mallock formula is incorrect and other models have to be used $[8,9]$. In the case of significant compressibility, the wave equation in the moving medium must be used instead of the Laplace equation:

$$
\Delta \Phi-\frac{1}{c_{e f f}^{2}}\left(\frac{\partial}{\partial t}+U \frac{\partial}{\partial z}\right)^{2} \Phi=0
$$

Equation (25) is written from the viewpoint of an immovable observer using a laboratory coordinate system. If the motion inside jet is described in a coordinate system connected to the jet and moving with the velocity $U$, it is necessary to put $U=0$ in (25).

One can seek for the solution of equation (22) and (25) in the form:

$$
\Phi=\sum_{m=0}^{\infty} D_{m}(\omega, k ; r) \exp (-i \omega t+i k z+i m \varphi) .
$$

The integer numbers $m$ indicates the angular modes. The zero mode $(m=0)$ corresponds to axially symmetric displacement; all cross-sections of the jet are circles which radii depend on time and distance $z$. Higher modes $(m=1,2,3, \ldots)$ describe displacements depending on the polar angle. Substituting (26) into (22) and (25) reduces the problem to:

$$
\begin{gathered}
\frac{d^{2} D_{m}}{d r^{2}}+\frac{1}{r} \frac{d D_{m}}{d r}-\left(k^{2}+\frac{m}{r^{2}}-\frac{\omega^{2}}{c_{e f f}^{2}}\right) D_{m}=0, \\
\left.\left(1-m^{2}-k^{2} R^{2}\right) \frac{d D_{m}}{d r}\right|_{r=R}+\frac{\rho_{L} R^{2}}{\sigma} \omega^{2} D_{m}(R)=0 .
\end{gathered}
$$

The solution of equation (27), satisfying the condition of being bounded inside the jet is expressed through the modified Bessel function:

$$
D_{m}(r)=C_{m} I_{m}\left(r \sqrt{k^{2}-\frac{\omega^{2}}{c_{e f f}^{2}}}\right) .
$$

Here, $C_{m}$ is an arbitrary constant. Substituting (30) into the dynamic boundary condition (22) in its form of (28) leads to the following dispersion law: 


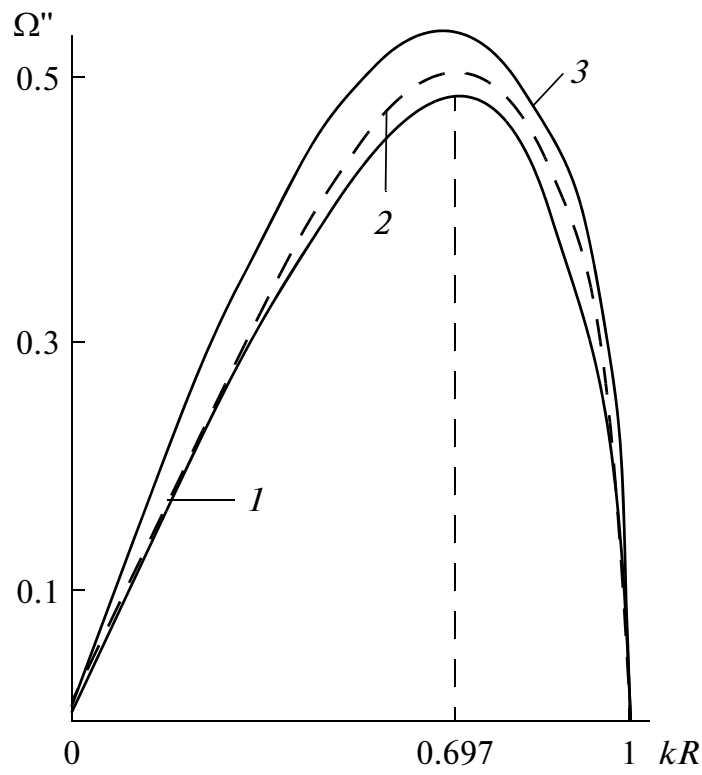

Fig. 2. Coefficient of instability of a water jet. Curve 1 corresponds to Rayleigh's theory [2]. Curve 2 (dashed) was calculated in [10]. Curve 3 demonstrates the effect of compressibility.

$$
\begin{gathered}
\left(1-m^{2}-k^{2} R^{2}\right) \sqrt{k^{2}-\frac{\omega^{2}}{c_{e f f}^{2}}} I_{m}^{\prime}\left(R \sqrt{k^{2}-\frac{\omega^{2}}{c_{e f f}^{2}}}\right) \\
+\frac{\rho_{L} R^{2}}{\sigma} \omega^{2} I_{m}\left(R \sqrt{k^{2}-\frac{\omega^{2}}{c_{e f f}^{2}}}\right)=0 .
\end{gathered}
$$

The prime here denotes the derivative with respect to the complete argument of the Bessel function. This formula generalizes the Rayleigh law (see [3], section 357 , formula (16)).

\section{INSTABILITY ANALYSIS}

Expression (31) is a complicated and implicit connection between the frequency $\omega$ and the wave number $k=2 \pi / \lambda$, where $\lambda$ is the wavelength of the disturbance on the jet surface. The frequency $\omega(k)$ determined by the dispersion law is a complex function:

$$
\omega(k)=\omega^{\prime}(k)+i \omega^{\prime \prime}(k) .
$$

The imaginary part, being substituted into (26), is responsible for exponential growth in time:

$$
\Phi=\sum_{m=0}^{\infty} D_{m} \exp \left[\omega^{\prime \prime}(m, k) t\right] \exp (i k z+\operatorname{im} \varphi) .
$$

So, if the imaginary part of the frequency is positive for some wave number, the jet is instable.

Consider, for simplicity, the zero (fundamental) mode and incompressible liquid. For this case the dispersion law (31) takes form:

$$
k R\left(1-k^{2} R^{2}\right) I_{1}(k R)+\frac{\rho_{L} R^{3}}{\sigma} \omega^{2} I_{0}(k R)=0 .
$$

The coefficient of instability then becomes:

$$
\Omega^{\prime \prime}=\omega " \sqrt{\frac{2 \rho_{L} R^{3}}{\sigma}}=\left[k R\left(1-k^{2} R^{2}\right) \frac{2 I_{1}(k R)}{I_{0}(k R)}\right]^{1 / 2} .
$$

As shown by Rayleigh, the jet is instable in the region $0<k R<1$, or $2 \pi R<\lambda<\infty$. Consequently, to cause instability the wavelength of a disturbance must be greater than the circumference of the cross-section of the jet. The dependence $\Omega^{\prime \prime}(k R)$ given by $(33)$ is shown by the solid curve 1 in Fig. 2. The result of [10] (published in 2000), which is less accurate than the law of [2] (published in 1878), is also shown in Fig. 2 by the dashed curve 2 . It differs from (33) by the factor:

$$
\frac{2 I_{1}(k R)}{k R \cdot I_{0}(k R)}
$$

present within the brackets of (33); it varies from 1 at $k R=0$ to approximately 0.89 at $k R=1$. The difference between [10] and [2] is insignificant in practical evaluations. One can easily see in (31) that for higher modes, the square of the frequency is positive. Consequently, the frequency has a zero imaginary part and the jet is stable.

Now the general form of the dispersion law (31) is analyzed for the fundamental mode. It is convenient to rewrite it in parametric form:

$$
\begin{gathered}
-\Omega^{2}(X)=X\left(1-X^{2}\right) \frac{2 I_{1}(X)}{I_{0}(X)}\left[1-\alpha X \frac{2 I_{1}(X)}{I_{0}(X)}\right]^{-1}, \\
k R=\sqrt{X^{2}+\alpha \Omega^{2}} .
\end{gathered}
$$

Here, $\Omega^{2}=\left(2 \rho_{L} R^{3} / \sigma\right) \omega^{2}$ is the square of the dimensionless frequency, and $\alpha=\sigma /\left(2 R \rho_{L} c_{e f f}^{2}\right)$ is a constant - a similarity parameter of the problem. Excluding parameter $X$ from the pair of equations, one can plot the curve $\Omega(k R)$ and its imaginary part as a function of the wave number or the wavelength. Curve 3, accounting for compressibility of the fluid, is also shown in Fig. 2 (with $\alpha=0.3$ ). As seen, compressibility increases the coefficient of instability. This means that the presence of bubbles inside the jet is undesirable.

Some wave resonance phenomena also exist [11, 12]. This affects the jet instability. Compressibility is also more significant at resonance. For example, the dispersion law with account for aerodynamic forces affecting the immovable asperity on the jet surface is:

$$
\begin{gathered}
\omega^{2}=-\frac{I_{1}(k R)}{I_{0}(k R)}\left[\frac{\sigma}{\rho_{L} R^{3}} k R\left(1-k^{2} R^{2}\right)\right. \\
\left.+\frac{\rho_{A}}{\rho_{L}} \frac{U^{2}}{R^{2} \beta} k^{2} R^{2} \frac{H_{0}^{(1)}(\beta k R)}{H_{1}^{(1)}(\beta k R)}\right], \beta=\sqrt{\frac{U^{2}}{c_{A}^{2}}-1 .}
\end{gathered}
$$

Here, $\rho_{A}, c_{A}$ are the density and the velocity of sound of air, respectively, $U$ is the velocity of the jet flow and $H_{0,1}^{(1)}$ are the Hankel cylindrical functions. One can see that if the jet velocity approaches the velocity of sound in air, the instability increases many times. Therefore, 
velocities close to the velocity of sound in air cannot be used in jet cutting technologies. Commonly, the velocity in practical applications is much higher than the velocity of sound of air. There also exist other resonances, giving restrictions on velocity and other key parameters of jets. These phenomena and corresponding regimes for the jet cutting process will be described in a coming paper.

\section{CONCLUSIONS}

The Rayleigh [2, 3] dispersion law is generalized and expressed more comprehensively and accurately than before; the law presented in this paper includes a new property - compressibility of the water due to air bubbles. It is shown how instability develops because of aerodynamic forces that appear at the streamlining of an initial irregularity of the equilibrium shape of the cross-section of the jet and how instability increases with increased concentration of gas bubbles, in accordance with experimental observations. It is also shown how resonance phenomena are responsible for strong instability. On the basis of the theoretical explanations given, conditions for stable operation are indicated. Comparison was made between the theory and existing experimental [13] and numerical studies [14]. The new theoretical results and improved understanding of the phenomenon of instability of high-speed water jets form a basis for the improvement of the water jet cutting technology. When polymer molecules are injected to improve stability of the jet, it is necessary to add the resulting elasticity of the fluid too into the dynamic boundary condition for the jet. Such generalization will be given in subsequent papers.

\section{ACKNOWLEDGMENTS}

Financial support from the Swedish Knowledge Foundation, Region Blekinge, Water Jet Sweden AB, GE Fanuc Automation CNC Nordic AB, Blekinge Institute of Technology, and Nizhni Novgorod State University (Mega-grant no. 11.G34.31.0066) is gratefully acknowledged.

\section{REFERENCES}

1. A. W. Momber, Principles of Abrasive Waterjet Machining (Springer Verlag, London, 1998).

2. J. W. S. Rayleigh, On the Instability of Jets, Proc. London Math. Soc. 29, 71 (1878).

3. J. W. S. Rayleigh, Theory of Sound (Dover, New York, 1945) Vol. 2.

4. L. D. Landau and E. M. Lifshitz, Hydrodynamics (Academic, New York, 1986; Glavredfizmatlit, Moscow, 1986).

5. H. Schlichting, Grenzschicht Theorie (Braun, Karlsruhe, 1951).

6. G. I. Broman and O. V. Rudenko, Phys.-Usp. 53, 91 (2010).

7. A. Mallock, Proc. Roy. Soc. London 84, 391 (1910).

8. V. E. Nakoryakov, B. G. Pokusaev, and I. R. Shreiber, Wave Propagation in Gas-Liquid Media (Energoatomizdat, Moscow, 1990; CRC, New York, 1993).

9. O. V. Rudenko and S. I. Soluyan, Theoretical Foundations of Nonlinear Acoustics (Plenum, New York, 1977).

10. Min Jou, J. Mater. Proc. Techn. 104, 17 (2000).

11. M. B. Vinogradova, O. V. Rudenko, and A. P. Sukhorukov, Theory of Waves (Nauka, Moscow, 1990).

12. A. A. Karabutov and O. V. Rudenko, Sov. Phys. Acoust. 25, 306 (1979).

13. P. Couty, Á. Spiegel, N. Vágó, B. I. Ugurta, and P. Hoffmann, Experiments in Fluids 36, 919 (2004).

14. P. B. Abrosimov, P. V. Zaplatin, V. S. Nagornyi, and A. A. Shmidt, Tech. Phys. 77, 1429 (2007). 